Ann. Biol. anim. Bioch. Biophys., I97I, 11 (I), I55-165.

\title{
DÉTERMINISME DU SEXE CHEZ LES HYBRIDES ENTRE TILAPIA MACROCHIR ET TILAPIA NILOTICA. ÉTUDE DE LA SEX-RATIO DANS LES RECROISEMENTS DES HYBRIDES DE PREMIERE GENERATION PAR LES ESPECES PARENTES
}

\author{
B. JALABERT *, P. KAMMACHER ** et P. LESSENT *** \\ * Station centrale de Physiologie animale, \\ Centre national de Recherches zootechniques, I.N.R.A., \\ 78 - Jouy-en-Josas \\ ** Faculté des Sciences d'Abidjan \\ *** Centre technique forestier tropical, \\ Station de Recherches piscicoles de Bouaké (Côte-d'Ivoire)
}

\section{RÉSUMÉ}

L'hypothèse d'une hétérogamétie mâle chez Tilapia nilotica et d'une hétérogamétie femelle chez Tilapia macrochir permet d'expliquer que les hybrides issus du croisement mâle macrochir $\times$ femelle nilotica sont tous mâles, alors que les hybrides issus du croisement réciproque ont une sex-ratio de trois mâles pour une femelle.

La présente étude se proposait de vérifier la validité de cette hypothèse en comparant ses conséquences théoriques avec les résultats expérimentaux relatifs à la sex-ratio des alevins de tous les rétrocroisements possibles : hybrides de première génération $\times$ parents.

Certaines contradictions dans ces résultats (concernant les 2 croisements réciproques: hybrides ( $\$$ nilotica $\times$ ㅇ macrochiv) $\times$ (nilotica) n'impliquent pas que l'hypothèse de départ soit fausse, mais obligent à faire quelques réserves sur la notion d'hétérochromosomes dans ces espèces.

\section{INTRODUCTION}

Les hybrides issus de certains croisements interspécifiques dans le genre Tilapia sont caractérisés par une sex-ratio anormale pouvant dans certains cas comporter Ioo p. Ioo de mâles. Les premières observations ont été effectuées en Malaisie (ANoN, I957-I959 et I959-I960; HiCKLING, I960) puis au Cameroun (BARD, I960) sur des espèces différentes. On doit rappeler que la production d'hybrides du seul sexe mâle chez Tilapia présente en pisciculture tropicale un très grand intérêt : l'élevage monosexe en étang évite la prolifération des alevins, facteur qui limitait jusqu'alors l'in- 
térêt économique de ces espèces. Les mâles chez les hybrides comme chez les espèces parentes ont une vitesse de croissance supérieure à celle des femelles (LESSENT, I966) ; enfin, les hybrides peuvent manifester une vigueur et des possibilités de croissance supérieures à celles des parents.

Ces hybrides tous mâles ont été obtenus jusqu'à présent dans les croisements suivants :

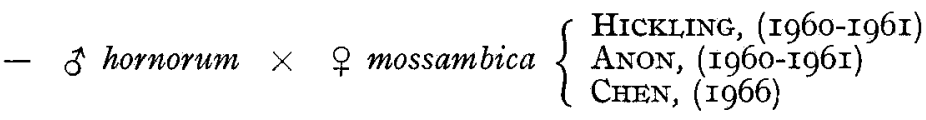

$$
\begin{aligned}
& \text { - }{ }^{*} \text { macrochir } \times \text { q nilotica } \quad\left\{\begin{array}{l}
\text { BARD, (I960) } \\
\text { LESSENT (I966) }
\end{array}\right. \\
& \text { - o hornorum } \times q \text { nilotica } \quad\left\{\begin{array}{l}
\text { LESSENT, (Ig } \\
\text { personnelle). }
\end{array}\right.
\end{aligned}
$$

Dans tous les cas, le croisement réciproque fournit $75 \mathrm{p}$. roo de mâles et $25 \mathrm{p}$. Ioo de femelles.

Une telle situation pose un intéressant problème de déterminisme du sexe. Dès I960, Hickling émettait 1'hypothèse d'un déterminisme génétique comparable à celui mis en évidence par GoRDON (I947) chez Platypoecilus où les croisements entre souches de différente origine donnent des résultats analogues; grâce à l'étude de la transmission de caractères de coloration liés au sexe, GoRdon a montré que le mâle est hétérogamétique $x y$ et la femelle homogamétique $x x$ dans certaines populations naturelles (région de Mexico), alors que la situation des chromosomes sexuels est inversée (femelles wy, mâles $y y$ ) dans une souche d'origine géographique différente (Honduras britannique).

Chez Tilapia, à notre connaissance, il n'a pas encore été mis en évidence de caractères simples liés au sexe ; cependant, une vérification moins immédiate de l'hypothèse est possible, par étude de la sex-ratio dans tous les recroisements possibles des hybrides avec les espèces parentes. Dans le cas de l'hybridation hornorum $\times$ mossambica, CHEN (I966 et I968) a donné un certain nombre de résultats de tels recroisements à partir des hybrides de première et deuxième génération $\left(F_{1}\right.$ et $\left.F_{2}\right)$.

Pour notre part, dans notre travail portant sur les hybrides macrochir $\times$ nilotica, nous nous sommes limités dans un premier temps à l'étude systématique de la sex-ratio des alevins de tous les rétrocroisements possibles hybrides $F_{1} \times$ parents.

\section{MATÉRIEL, ET MÉTHODES}

Toute l'expérimentation a été effectuée à la station de pisciculture du Centre technique forestier tropical de Bouaké (Côte-d'Ivoire) depuis 1965. La souche de Tilapia nilotica utilisée est originaire du bassin de la Volta noire, celle de T. macrochir du Cameroun où l'espèce avait été introduite à partir du Katanga. Les hybrides produits (Lessent, I966) appartiennent aux deux groupes respectivement issus des deux croisements réciproques :

I. $\sigma^{2}$ macrochir $\times$ o nilotica $\rightarrow$ Hybrides (I) (I00 p. Ioo $\delta$ )

II. $\delta$ nilotica $\times \stackrel{+}{+}$ macrochir $\rightarrow$ Hybrides (II) $(75$ p. roo $\delta+25$ p. 100 우 $)$ 

sibles :

A partir de ces hybriđes, ont été réalisés systématiquement tous les rétrocroisements pos-

$\begin{array}{cll}\text { Rétrocroisement } & \\ \text { - } & & \\ \text { A } & \text { o Hybride (I) } & \times \\ \text { B } & \text { o Hybride (I) } & \times \\ \text { C } & \text { o Hybride (II) } & \times \\ \text { D } & \text { o Hybride (II) } & \times \\ \text { E } & \text { o nilotica } & \times \\ \text { F } & \text { o macrochir } & \times\end{array}$

Nombre de.

répétitions

$\begin{array}{ll}\text { o macrochir } \\ \text { o nilotica } \\ \text { o } \\ \text { nilotica } \\ \text { o macrochir } \\ \text { o } & \text { Hybride (II) } \\ \text { o } & \text { Hybride (II) }\end{array}$

-
3
2
8
8
8
8

Chaque répétition est constituée par la progéniture d'un couple différent. Chaque couple a été placé dans un étang de $50 \mathrm{~m}^{2}$; deux mois après l'apparition des premiers alevins, ceuxci ont été transférés dans des étangs d'élevage de $400 \mathrm{~m}^{2}$ et sexés à l'âge de 4 à 6 mois, après différentiation externe apparente des sexes.

\section{RÉSULTATS}

Les résultats des différents rétrocroisements sont consignés dans les tableaux $\mathrm{A}$ à $F$. Admettons que dans les deux espèces macrochir et nilotica des facteurs féminisants et masculinisants essentiels sont portés respectivement par les hétérochromosomes de type $\mathrm{X}$ et $\mathrm{Y}$.

Nous nous proposons de comparer nos résultats expérimentaux aux résultats théoriques découlant de 1'hypothèse d'une hétérogamétie mâle chez Tilapia nilotica et d'une hétérogamétie femelle chez $T$. macrochir. Cette hypothèse nous conduit à écrire comme suit les formules chromosomiques des sexes dans les deux espèces:

$\begin{array}{ccc} & \text { T. nilotica } & \text { T. macrochir } \\ \text { Mâle } & \overline{\mathrm{Y}} & - \\ \text { Femelle } & x x & y y \\ \mathrm{X} y\end{array}$

Cette formulation qui facilite la confrontation des génotypes dans les deux espèces, implique que $\mathbf{Y}$ porteur de facteurs masculinisants déterminants domine $x$ chez $T$. nilotica, alors que chez $T$. macrochir, $\mathrm{X}$ est dominant sur $y$.

Avec ces conventions, et en admettant que les 4 chromosomes notés $x, \mathrm{Y}, \mathrm{X}, y$ sont homologues entre eux, la constitution génétique de la première génération d'hybrides est la suivante :

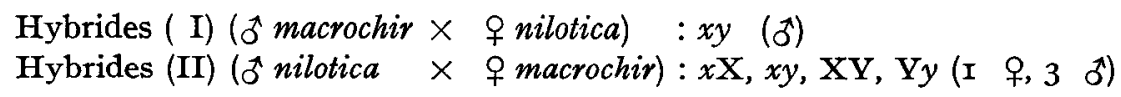

Si nous admettons encore que dans la confrontation des chromosomes sexuels des deux espèces, $y$ est déterminant face à $x$, et $\mathrm{Y}$ déterminant face à $\mathrm{X}$, le seul génotype de 1'hybride (I), xy est mâle, alors que les 4 génotypes des hybrides (II) correspondent à 3 mâles pour une femelle, ce qui est vérifié par les faits expérimentaux (voir plus haut).

Examinons les répercussions de cette théorie sur les résultats des rétrocroisements. 
TABLEAU A

(Rétrocroisement A) Descendance de 3 couples: ot hybride (I) $\times$ ㅇ macrochir

\begin{tabular}{|c|c|c|c|c|c|c|}
\hline \multirow{2}{*}{ No } & \multicolumn{2}{|c|}{$\begin{array}{l}\text { Nombre d'alevins } \\
\text { sexés }\end{array}$} & \multicolumn{2}{|c|}{$\begin{array}{l}\text { Pourcentage } \\
\text { des sexe; }\end{array}$} & \multirow{2}{*}{$\begin{array}{c}\text { Intervalle de confiance } \\
\text { du pourcentage de }+ \\
\text { au seuil } p=95\end{array}$} & \multirow{2}{*}{$\begin{array}{l}\text { Rapport } \\
\text { théorique } \\
\text { simple } \\
\text { possible }\end{array}$} \\
\hline & o & 우 & $\sigma^{*}$ & 우 & & \\
\hline$A_{1}$ & 187 & 192 & 49,3 & 50,7 & $45,4<\% \quad q<55,6$ & $1 / 1$ \\
\hline $\mathrm{A}_{2}$ & 167 & 182 & 47,9 & 52,1 & $46,7<\% \quad$ o $<57,5$ & $1 / 1$ \\
\hline$A_{9}$ & 183 & 177 & 50,8 & 49,2 & $43,8<\%$ 安 $<54,5$ & $1 / 1$ \\
\hline
\end{tabular}

TABLEAU B

(Rétrocroisement B) Descendance de 2 couples: ô hybride (I) $\times \&$ nilotica

\begin{tabular}{|c|c|c|c|c|c|c|}
\hline \multirow{2}{*}{ No } & \multicolumn{2}{|c|}{$\begin{array}{c}\text { Nombre d'alevins } \\
\text { sexés }\end{array}$} & \multicolumn{2}{|c|}{$\begin{array}{l}\text { Pourcentage } \\
\text { des sexes }\end{array}$} & \multirow{2}{*}{$\begin{array}{l}\text { Intervalle de confiance } \\
\text { du pourcentage de } \\
\text { au seuil } p=95\end{array}$} & \multirow{2}{*}{$\begin{array}{c}\text { Rapport } \\
\text { théorique } \\
\text { simple } \\
\text { possible }\end{array}$} \\
\hline & 0 & 운 & $\sigma^{*}$ & 우 & & \\
\hline $\mathrm{B}_{1}$ & 195 & 172 & 53,1 & 46,9 & $41,6<\% \quad$ Q $<52,1$ & $1 / 1$ \\
\hline $\mathrm{B}_{2}$ & 162 & 200 & 44,6 & 55,4 & $50,0<\% \quad q<60,5$ & $1 / 1$ \\
\hline
\end{tabular}




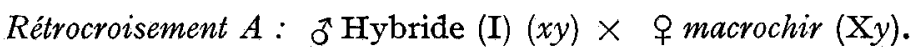

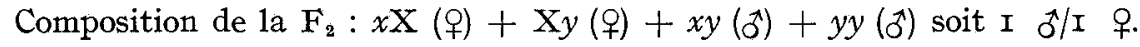

La sex-ration attendue est de $I$ ô pour I $q$ ce qui est conforme à l'expérience (tabl. A).

Rétrocroisement $B$ : $\hat{0}$ Hybride (I) $(x y) \times$ ㅇ nilotica $(x x)$.

Composition de la $\mathrm{F}_{2}: x x\left(\right.$ ( ) $+x y\left(\sigma^{*}\right)$ soit $\mathrm{I} \quad \delta^{\star} / \mathrm{I}$ 우

La sex-ratio attendue I $\delta / I$ \& est encore conforme à l'expérience (tabl. B).

Rétrocroisement $C$ : o Hybride (II) (xy ou XY ou Yy) $\times$ 우 nilotica $(x x)$.

Suivant le génotype du $\delta$ hybride (II), il devrait y avoir 3 possibilités de $F_{2}$ :

a) avec of $x y: x x($ (q) $+x y(\delta)$, soit I $x / I$ \&

b) avec $\hat{\sigma} \mathrm{XY}: x \mathrm{Y}(\hat{\jmath})+x \mathrm{X}($ (q) $)$, soit I $\delta / \mathrm{I}$ 우

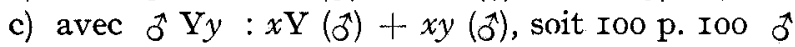

Aucune de ces distributions théoriques ne se retrouve dans les résultats expérimentaux (tabl. C).

Rétrocroisement D: đิ Hybride (II) ( $x y$, ou XY ou $\mathrm{Y} y) \times q$ macrochir $(\mathrm{X} y)$.

Dans ce cas aussi, il devrait y avoir 3 possibilités de $F_{\mathbf{2}}$.

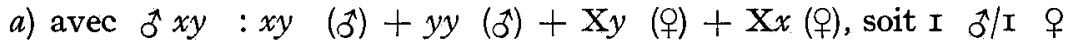
b) avec $\delta \mathrm{XY}: \mathrm{XY}(\delta)+\mathrm{Yy}(\delta)+\mathrm{XX}($ o $)+\mathrm{X} y($ () $)$, soit I $\delta / \mathrm{I}$ 우

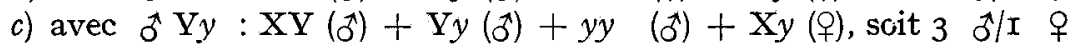

La comparaison avec les résultats du tableau $\mathrm{D}$ montre que la répartition I $\hat{\delta} / \mathrm{I}$ q est vérifiée par les rétrocroisements $\mathrm{D}_{3}$ et $\mathrm{D}_{4}$, et la répartition : $3 \hat{\delta} / \mathrm{I}$ 우 par $\mathrm{D}_{1}, \mathrm{D}_{2}, \mathrm{D}_{6}$ et $\mathrm{D}_{7}$.

Parmi les génotypes de la $\mathrm{F}_{2}$ théorique $(b)$ ci-dessus, nous trouvons la combinaison nouvelle $\mathrm{XX}$. Si cette combinaison n'était pas viable, la sex-ratio théorique de $(b)$ serait $2 \delta / I$ q ce qui correspondrait alors au résultat du rétrocroisement $D_{8}$.

Rétrocroisement $E$ : đ̛ nilotica $(x \mathrm{Y}) \times$ \& hybride (II) $(x \mathrm{X})$.

Composition de la $\mathrm{F}_{2}: x \mathrm{Y}\left({ }^{\prime}\right)+\mathrm{XY}(\widehat{)})+x x($ o $)+x \mathrm{X}($ (ᄋ) $)$ soit $\mathrm{I} \delta / \mathrm{I}$ 우.

Nous ne retrouvons cette répartition théorique dans aucun des résultats du tableau E.

Rétrocroisement $F$ : đo macrochir $(y y) \times$ ㅇ Hybride (II) $(x \mathrm{X})$.

Compositicn de la $\mathrm{F}_{2}: x y\left(\delta^{*}\right)+\mathrm{X} y\left(\right.$ q), soit $\mathrm{I} \quad \delta^{x} / \mathrm{I}$ 우.

Cette sex-ratio se retrouve dans les rétrocroisements $F_{1}, F_{4}, F_{8}$ et est approchée dans $\mathrm{F}_{5}$. 


\section{TABLEAU C}

(Rétrocroisement C) Descendance de 8 couples : ô hybride (II) $\times$ 우 nilotica

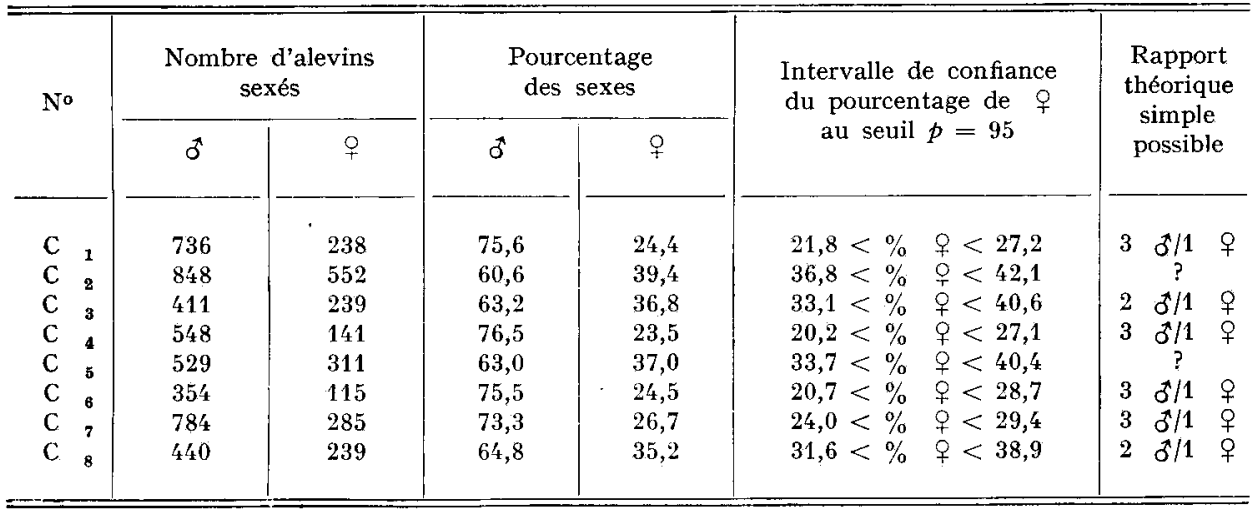

\section{TABLEAU D}

(Rétrocroisement D) Descendance de 8 couples : ơ hybride (II) $\times$ o macrochir

\begin{tabular}{|c|c|c|c|c|c|c|}
\hline \multirow[t]{2}{*}{ No } & \multicolumn{2}{|c|}{ Nombre d'alevins sexés } & \multicolumn{2}{|c|}{$\begin{array}{c}\text { Pourcentage } \\
\text { des sexes }\end{array}$} & \multirow{2}{*}{$\begin{array}{c}\text { Intervalle de confiance } \\
\text { du pourcentage de } \\
\text { au seuil } p=95\end{array}$} & \multirow{2}{*}{$\begin{array}{c}\text { Rapport } \\
\text { théorique } \\
\text { possible } \\
\text { simple }\end{array}$} \\
\hline & $\delta$ & ㅇ․ & $\sigma$ & $q$ & & \\
\hline $\begin{array}{l}\mathrm{D}_{1} \\
\mathrm{D}_{2} \\
\mathrm{D}_{3} \\
\mathrm{D}_{4}\end{array}$ & $\begin{array}{r}250 \\
202 \\
29 \\
187\end{array}$ & $\begin{array}{r}91 \\
74 \\
28 \\
167\end{array}$ & $\begin{array}{l}73,3 \\
73,2 \\
50,9 \\
52,8\end{array}$ & $\begin{array}{l}26,7 \\
26,8 \\
49,1 \\
47,2\end{array}$ & $\begin{array}{ll}22,0<\% & \text { q }<31,7 \\
21,7<\% & \text { 우 }<32,5 \\
35,7<\% & \text { ㅇ }<62,5 \\
\mathbf{4 1 , 5}<\% & \text { q }<52,9\end{array}$ & $\begin{array}{lll}3 & \delta / 1 & \text { + } \\
3 & \delta / 1 & + \\
1 & \delta / 1 & + \\
1 & \delta / 1 & 9\end{array}$ \\
\hline $\mathrm{D}_{5}$ & & & & de rep & ion & \\
\hline $\begin{array}{l}D_{6} \\
D_{7} \\
D_{8}\end{array}$ & $\begin{array}{l}380 \\
260 \\
196\end{array}$ & $\begin{array}{r}98 \\
89 \\
108\end{array}$ & $\begin{array}{l}79,5 \\
74,5 \\
64,5\end{array}$ & $\begin{array}{l}20,5 \\
25,5 \\
35,5\end{array}$ & $\begin{array}{ll}17,0<\% & \text { ㅇ }<24,4 \\
21,0<\% & \text { \& }<30,4 \\
30,1<\% & \text { q }<41,2\end{array}$ & $\begin{array}{lll}3 & \delta / 1 & \text { } \\
3 & \delta / 1 & \text { ㅇ } \\
2 & \delta / 1 & +\end{array}$ \\
\hline
\end{tabular}


TABI,EAU E

(Rétrocroisement E) Descendance de 8 couples : $\sigma^{\star}$ nilotica $\times$ ㅇ hybride (II)

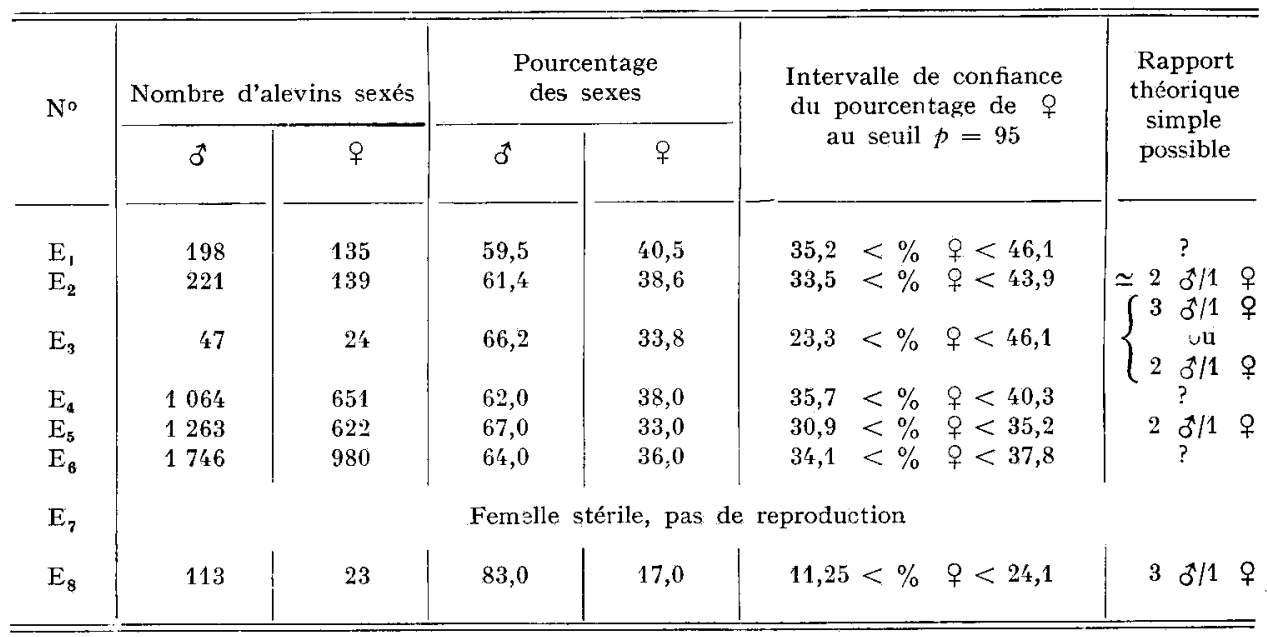

TABLEAU F

(Rétrocroisement F) Descendance de 8 couples : $\delta$ macrochir $\times \&$ hybride (II)

\begin{tabular}{|c|c|c|c|c|c|c|c|}
\hline \multirow{2}{*}{ No } & \multicolumn{2}{|c|}{ Nombre d'alevins sexés } & \multicolumn{2}{|c|}{$\begin{array}{l}\text { Pourcentage } \\
\text { des sexes }\end{array}$} & \multirow{2}{*}{\multicolumn{2}{|c|}{$\begin{array}{c}\text { Intervalle de confiance } \\
\text { du pourcentage de } \\
\text { au seuil } p=95\end{array}$}} & \multirow{2}{*}{$\begin{array}{l}\text { Rapport } \\
\text { théorique } \\
\text { simple } \\
\text { possible }\end{array}$} \\
\hline & $\sigma^{\pi}$ & 9 & $\sigma^{*}$ & 우 & & & \\
\hline $\begin{array}{l}\mathrm{F}_{1} \\
\mathrm{~F}_{2}\end{array}$ & $\begin{array}{l}165 \\
157\end{array}$ & $\begin{array}{r}126 \\
79\end{array}$ & $\begin{array}{l}56,7 \\
66,5\end{array}$ & $\begin{array}{l}43,3 \\
33,5\end{array}$ & $\begin{array}{l}37,5<\% \\
27,4<\%\end{array}$ & 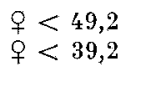 & $\simeq \begin{array}{lll}1 & \delta / 1 & + \\
2 & \delta / 1 & 0\end{array}$ \\
\hline $\mathrm{F}_{3}$ & \multicolumn{7}{|c|}{ Femelle stérile, pas de reproduction } \\
\hline $\begin{array}{l}F_{4} \\
F_{5}\end{array}$ & $\begin{array}{l}969 \\
658\end{array}$ & $\begin{array}{l}883 \\
557\end{array}$ & $\begin{array}{l}52,3 \\
54,2\end{array}$ & $\begin{array}{l}47,7 \\
45,8\end{array}$ & $\begin{array}{l}45,4<\% \\
43,0<\%\end{array}$ & $\begin{array}{l}9<50,0 \\
+9<48,7\end{array}$ & $1 \underset{?}{0 / 1}+9$ \\
\hline$F_{6}$ & \multicolumn{7}{|c|}{ Femelle stérile, pas de reproduction } \\
\hline $\begin{array}{l}F_{7} \\
F_{8}\end{array}$ & $\begin{array}{l}131 \\
369\end{array}$ & $\begin{array}{l}207 \\
327\end{array}$ & $\begin{array}{l}38,8 \\
53,0\end{array}$ & $\begin{array}{l}61,2 \\
47,0\end{array}$ & $\begin{array}{l}55,8<\% \\
43,2<\%\end{array}$ & 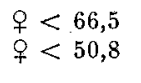 & $1 \stackrel{?}{\delta / 1}$ 우 \\
\hline
\end{tabular}




\section{DISCUSSION}

Cette étude apporte des éléments d'information contradictoires. L'observation des sex-ratios dans la première génération $\left(\mathrm{F}_{1}\right)$ des croisements réciproques entre Tilapia nilotica et $T$. macrochir nous a conduits à imaginer chez ces poissons un méca . nisme précis de détermination du sexe fondé sur l'existence d'un système d'hétérochromosomes, avec hétérogamétie mâle chez $T$. nilotica et hétérogamétie femelle chez T. macrochir, sachant par ailleurs que la sex-ratio dans chacune de:; deux espèces pures élevées à la station de Bouaké est sensiblement de I. Or, l'analyse systématique de tous les croisements en retour des hybrides $\left(F_{1}\right)$ par les espèces parentes montre que certains résultats sont en désaccord avec la théorie énoncée ci-dessus. En effet, si les résultats expérimentaux des rétrocroisements $A, B, D$, et $F$ tendent à confirmer la théorie, les résultats des croisements réciproques $\mathrm{C}$ et $\mathrm{E}$, (hybride $(\hat{o}$ nilotica $\times$ q macrochir $)) \times$ nilotica sont complètement différents des sex-ratios attendues et ne peuvent s'expliquer par l'effet des hétérochromosomes seuls.

Ces observations sont à rapprocher des travaux effectués par F. Y. CHEN (I966, 1968) sur l'hybridation entre Tilapia hornorum et $T$. mossambica. Dans ce cas également, l'hypothèse d'une hétérogamétie mâle chez l'une des espèces parentes (mossambica) et femelle chez l'autre (hornorum) ne suffit pas à expliquer la totalité des faits constatés quant à la sex-ratio dans des populations obtenues par des modes de filiation variés. Les croisements réciproques : hybride (ô mossambica $\times$ q hornorum) $\times$ mossambica ne semblent pas avoir été inclus dans cette série d'expériences génétiques, ce qui est dommage, car ils auraient constitué le pendant de nos croisements : hybride (Jnilotica $\times \subsetneq$ macrochir $) \times$ nilotica où la sex-ratio n'obéit pas au mécanisme supposé. Enfin, certains croisements entre hybrides de deuxième génération et espèces parentes ont donné des résultats surprenants que $F$. $Y$. ChEN a expliqué par une mortalité différentielle de certains génotypes.

Le rapprochement de ces données montre que le déterminisme génétique du gonochorisme chez les hybrides d'espèces de Tilapia cesse apparemment de se présenter d'une manière simple dès que le stade de la $F_{1}$ est dépassé. Il convient donc de s'interroger sur la validité de la notion d'hétérochromosomes en ce qui concerne ce genre, tout en émettant quelques réserves sur le critère d'analyse utilisé, c'est-à-dire l'observation tardive de la sex-ratio, à l'âge de 4 à 6 mois.

Chez les Poissons, la preuve cytologique de l'existence d'hétérochromosomes sexuels n'a été établie jusqu'à présent avec certitude que chez quelques espèces, parmi lesques Bathylagus wesethi et Gambusia affinis (CHEN et EBEIING, I966, I968; CHEN, T. R. I969; EBELING et CHEN, I970). Mais généralement les preuves d'une digamétie sexuelle sont d'ordre purement génétique, fournies grâce à certains caractères simples de coloration liés au sexe, par l'étude de la descendance d'individus de phénotype sexuel inverse du génotype sexuel présumé (travaux revus par T. Yamamoto, I969). Dans le genre Tilapia, Clemens et InsleE (I968) ont mis en évidence une homogamétie femelle chez $T$. mossambica (conforme, donc, à une partie de l'hypothèse de $F$. Y. CHEN) après avoir réalisé des inversions sexuelles fonctionnelles de femelles en mâles par action de la méthyltestostérone incorporée à l'alimentation de jeunes alevins à un stade indifférencié. 
Dans notre propre étude, des observations préliminaires ont montré que $T$. nilotica et $T$. macrochir ainsi que leurs hybrides possèdent tous un caryotype identique $(2 n=44)$ où la présence de chromosomes hétéromorphes liés au sexe n'est pas évidente. D'autre part, nous ne connaissons pas chez les deux espèces étudiées, de caractères simples liés au sexe. C'est pourquoi, en première analyse, nous avons cherché à vérifier l'existence d'un système XX, XY, par l'étude de la sex-ratio de diverses population hybrides. Il est certain que ce procédé peut conduire à des résultats ambigus, car le sexage pratiqué sur des poissons âgés de quatre à six mois ne reflète que de loin ce que BACCI (I965) appelle la sex-ratio primaire, c'est-à-dire la proportion de mâles et de femelles au moment de la fécondation, élément à prendre fondamentalement en considération dans l'analyse formelle de notre problème. Des phénomènes de mortalité sélective peuvent intervenir à diverses étapes du développement, depuis les premiers stades embryonnaires jusqu'après la différenciation sexuelle totale, de sorte que la sex-ratio observée par sexage d'une pcpulation adulte n'a qu'une valeur relative.

Une telle possibilité n'est pas à rejeter a priori, comme l'a d'ailleurs constaté F. Y. CHEN (I969) chez les hybrides entre Tilapia hornorum et $T$. mossambica, mais elle demanderait à être vérifiée par un sexage histologique aussi précoce que possible, sur des alevins ou même des embryons, ce qui impliquerait en outre l'étude préalable de la période et du mode de différenciation du sexe au cours du développement de ces animaux.

Il faut aussi prendre en considération l'éventualité que d'autres phénomènes étrangers à l'hypothèse simple de départ peuvent altérer la sex-ratio prévue au cours des filiations successives d'hybrides interspécifiques : divers systèmes de détermination génétique du sexe ont été mis en évidence c'łez les Poissons gonochoriques (travaux revus par GoRdoN, I957 ; BACCr, I965; YAMAMOTO, I969) depuis le système dit de «détermination polyfactorielle du sexe » de Xiphophorus helleri où gènes masculinisants et féminisants sont répartis sur les autosomes en des équilibres variés induisantle phénotype mâle ou femelle (Kosswig et coll., I933 à I964), jusqu'aux systèmes mettant en jeu un couple de chromosomes sexuels avec hétérogamétie mâle ou femelle. Mais même dans ce cas, la correspondance entre phénctype sexuel et génotype sexuel supposé d'après la présence ou l'absence de l'hétérochromosome n'est pas forcément rigoureuse. En effet, les travaux de WrNGE (I923 à I948) ont montré que les chromosomes sexuls sont peu différenciés chez les Lebistes reticulatus, et que des gènes portés par les autosomes peuvent assumer tune part importante dans la réalisation du phénotype sexuel. Il en est de même chez Oryzias latipes chez lequel, d'après Yamamoto ( 1969 ) le sexe est bien déterminé par le jeu des chromosomes sexuels, à l'exception toutefois de certains individus dont le phénotype sexuel semble devoir s'expliquer non seulement par le jeu des chromosomes sexuals, mais aussi par la recombinaison d'autosomes qui créarait d'una manière fortuite des associations de gènes susceptibles de jouer un rôle dans le déterminisme du sexe. Une telle situation est vraisemblablement assez répandue chez les Téléostéans gonochoriques et on peut penser qu'elle prévaut aussi dans le genre Tilapia. Par conséquent, dans une étude de la sex-ratio chez les hybrides d'espèces à génomes très voisins, comme c'est le cas ici, il faut tenir compte de la réalité d'une recombinaison affectant non seulement les chromosomes porteurs chaz les parents de l'information qui détermine le sexe, mais l'ensemble des autosomes. Du brassage résultant de cet apparie- 
ment hétérogénétique entre les chromosomes homologues de $T$. nilotica et $T$. macrochi peuvent naître des associations de gènes dont l'action et l'inter-action sont susceptibles de jouer un rôle dans le déterminisme du sexe en altérant le mécanisme des systèmes en jeu dans les espèces pures. Même si la digamétie sexuelle prévaut à la première génération hybride, la néoformation d'autres systèmes de détermination du sexe est possible par la suite. Une sélection peut alors favoriser des associations géniques complexes où le cumul des fonctions de gènes autosomaux vient se superposer à l'activité des gènes supérieurs localisés sur les chromosomes sexuels.

Rien ne s'oppose donc à considérer que les Tilapia sont caractérisés par un mode de détermination du sexe relativement simple au départ, mais où la digamétie propre aux espèces pures peut non seulement se renverser d'une unité systématique à une autre, mais encore être relayée par une " polygamétie " d'origine autosomale, en particulier dans la ségrégation des hybrides interspécifiques. A cet égard, la situation observée dans les croisements du type : hybride (o nilotica $\times$ q macrochir) $\times$ nilotica mériterait d'être fouillée plus avant par de nouvelles études génétiques et caryologiques.

En attendant d'autres preuves expérimentales de cet ordre, nous retiendrons momentanément l'hypothèse de la coexistence dans le genre Tilapia d'espèces à hétérogamétie mâle et d'espèces à hétérogamétie femelle, tout en admettant que les chromosomes sexuels en jeu dans les espèces pures n'ont qu'une valeur déterminante toute relative, susceptible d'être contrebalancée par des facteurs masculinisants et féminisants portés par des autosomes.

Rę̧u pour publication en novembre 1970.

\section{SUMMARY}

\section{SEX DETERMINISM IN TILAPIA MACROCHIR X TILAPIA NILOTICA HYBRIDS INVESTIGATION ON SEX RATIO IN IST GENERATION HYBRID $\times$ PARENT CROSSINGS}

Male heterogamety in Tilapia nilotica and female heterogamety in Tilapia macrochir have been tentatively postulated to account for the fact that all $\delta$ macrochir $\times$ o nilotica hybrids are male, whereas the sex ratio of the reciprocal crossing is 3 male to $I$ female hybrids.

The current study was undertaken to further substantiate this hypothesis by comparing its theoretical models with experimental results obtained for all possible Ist generation hybrid $\times$ parent crossings.

Some contradictory results concerning the two reciprocal crossings : o nilotical o macrochir hybrids $\times$ nilotica are not preclusive of the hypothesis, but lead us to make some reservations on the concept of heterochromosomes for these species.

\section{RÉFÉRENCES BIBLIOGRAPHIQUES}

Anon, 1957-59; 1959-1960; I960-196r. Annual Report of Tropical fish culture Research Institute. Batu Berendam Malacca. Malaisie.

Bacci G., I965. Sex determinism. Pergamon Presss $306 \mathrm{pp}$.

BARD J., I96o. Hybridation des Tilapia. C. R. Symp. Lusaka, publ. C. S. A., n० 63. I79-I82.

EHEN F. Y., 1966. Preliminary studies on the sex-determining mechanism of Tilapia mossambica PETERS and Tilapia hornorum trewavas. Annual. Rep. Trop. Fish Cult. Inst. Batu Berendam Malacca. Appendix IV, 43-47. 
Chen F. Y., I968. Further studies on the sex-determining mecanism of Tilapia mossambica PETERS and Tilapia hornorum trewavas. Annual Rep. Trop. Fish Cult. Inst. Batu Berendam Malacca, Appendix I, 25-29.

Chen F. Y., I969. Preliminary studies on the sex-determining mechanism of Tilapia mossambica PETERS et Tilapia hornorum trewavas. Verh. Internat. Verein. Limnol., 17, 719-724.

Chen T. R., r969. Karyological heterogamety of deep-ssa fishes. Postilla 13029 p.

Chen T. R., Ebeling A. W., I966. Probable male heterogamety in the deep-sea fish Bathylagus wesethi (Teleostei, Bathylagidae). Chromosoma (BERL), 18, 88-96.

Chen T. R., Ebeling A. W., 1968. Karyological evidence of female heterogamety in the mosquito fish. Gambusia affinis. Copeia, I968 (x), 70-75.

Clemens H. P., Inslee T., 1968. The production of unisexual broods by Tilapia mossambica sexreversed with methyltestosterone. Trans. Amer. Fish. Soc., 97, I8-21.

Ebeling H. W.; Chen T. R., 1970. Heterogamety in teleostean fishes. Trans. Amer. fish. Soc., 99, I3I-I38.

GoRdon M., 1947 a. Genetics of Platypoecilus maculatus. IV. The sex-determining mechanism in two wild populations of the mexican platyfish. Genetics, 38, 8 .

Gordon M., I95I. Genetics of Platypoecilus maculatus. V. Heterogametic sex-determining mechanism in females of domesticated stock originally from British Honduras. Zoologica. 36, 127-I 34.

Gordon M., I957. Physiological genetics of fishes, in Physiology of Fishes Ed. Brown M. E. Academic Press Inc. New York. Vol. 2, 43r-5or.

Gordon M., Aronowitz O., I95I. Sex-determination in Platypoecilus maculatus. II. History of a male platyfish that sired all female broods. Zoologica, 36, I47-I53.

Hickling C. F., r960. The Malacca Tilapia hybrids. J. Genet., 57, I-ro.

KosswiG C., I933. Genotypiche and phänotypische Geschlechtbestimmung bei Zahnkarpfen. II. Biol. Zbl., 53, 752-759. (cité par Kosswig, I936).

Kosswig C., I935-1936. Die Kreutzung zweier XX bzw. XY-Geschlechter miteinander und der Ersatz eines Y-chromosoms einer Art durch das X-chromosomeiner Anderen. Züchter, 78, 40-48.

Kosswig C., I936. Nicht homologe Heterochromosomen bei nachstverwandten Arten (Kreutzungen von Platypoecilus maculatus und Pl. sciphidium). Biol. Zentr., 56, I99-207.

Kosswig C., OKтаY M., 1939. Geschlechtbestinmung in Kreuzungen zwischen Xiphophorus und Platypoecilus. C. R. Fac. Sci., Istambul, 4, I-54.

Kosswig G., 1964. Polygenic sex determination. Experientia, 20, 190-199.

Lessent P., I966. Essais d'hybridation dans le genre Tilapia à la station de Recherches piscicoles de Bouaké, Côte-d'Ivoire. F. A. O. Fish Rep., 44 (4), 148-159.

WINGE O., 1922. A peculiar mode of inheritance and its cytological explanation. J. Genet., 12, I37-r44.

WINGE O., 1923. Grossing-over between X and Y chromosomes in Lebistes. J. Genet., 13, $201-227$.

WINGE O., I930. On the occurrence of $x x$ males in Lebistes. J. Genet., 23, 69-76.

WINGE O., I934. The experimental alteration of sex chromosomes into autosomes (and vice versa) as illustrated by Lebistes. C. R. Lab. Carlsberg (Ser. Physiol.), 21, 1-49.

Winge O., Ditlevsen E., 1948. Colour inheritance and sex determination in Lebistes. C. R. Lab. Carlsberg (Ser. Physiol.), 24, 227-248.

Yамамото T., 1969. Sex differentiation, in Fish Physiology Ed. Hoar D. J., and Randall W. S. Academic Press, New York, Vol. 3, I I7-I 75. 